\title{
Application of an indocyanine green-mediated fluorescence imaging navigation system in detecting mice tumors
}

\author{
Xueyan Zhao ${ }^{1}$, Ziyu Wang ${ }^{1}$, Yulin $W^{2}{ }^{2}$, Huiming Cai ${ }^{2}$ \\ ${ }^{1}$ School of Basic Medicine and Clinical Pharmacy, China Pharmaceutical University, Nanjing, China; ${ }^{2}$ Nanjing Nuoyuan Medical Devices Co., Ltd., \\ Nanjing, China \\ Contributions: (I) Conception and design: H Cai; (II) Administrative support: H Cai; (III) Provision of study materials or patients: Y Wu; (IV) \\ Collection and assembly of data: X Zhao, Z Wang; (V) Data analysis and interpretation: X Zhao, Z Wang; (VI) Manuscript writing: All authors; (VII) \\ Final approval of manuscript: All authors. \\ Correspondence to: Huiming Cai; Yulin Wu. Nanjing Nuoyuan Medical Devices Co., Ltd., Fuqian South Road 18, Nanjing 211514, China. \\ Email: chm9166@aliyun.com; wylcpu0801@163.com.
}

Background: Surgical operation plays an important role in the treatment of cancer. The success of the
operation lies in the complete removal of the primary and disseminated tumor tissue while preserving the
normal tissue. The development of optical molecular image navigation technology has provided a new option
for intraoperative tumor visualization. In this study, a fluorescence imaging navigation system was used to
detect the diameter of mice tumors and provide experimental evidence for the further development of digital
diagnosis and treatment equipment. Methods: The minimum detection concentration in vitro of the fluorescence imaging navigation system for indocyanine green (ICG) was first detected, then 120 female Institute of Cancer Research (ICR) mice and 120 female BALB/c nude mice were randomly divided into three groups by weight, high-dose ( $\mathrm{H}, 4 \mathrm{mg} / \mathrm{kg})$, middle-dose (M, $2 \mathrm{mg} / \mathrm{kg}$ ), and low-dose (L, $1 \mathrm{mg} / \mathrm{kg}$ ) groups of ICG solution. After inoculating solid tumors, high, medium, and low doses of ICG were injected via the tail vein, and the tumor diameter was measured by a fluorescence imaging navigation system and vernier caliper within 24 hours of injection.

Results: The minimum detectable diameter of the system could reach $0.2 \mathrm{~mm}$ compared with the vernier caliper, and the actual measurement error was within $0.2 \mathrm{~mm}$.

Conclusions: A fluorescence imaging navigation system has high accuracy and sensitivity in the application of tumor detection, which may assist the clinical diagnosis and treatment of tumors.

Keywords: Indocyanine green (ICG); cancer; fluorescence imaging navigation system; fluorescence imaging

Submitted May 25, 2021. Accepted for publication Jul 14, 2021.

doi: 10.21037/atm-21-3050

View this article at: https://dx.doi.org/10.21037/atm-21-3050

\section{Introduction}

Cancer causes nearly 10 million deaths globally on an annual basis (1) and imposes a huge economic burden on society $(2,3)$. The treatment of cancer is often difficult, and current methods include surgery, radiotherapy, systemic therapy, interventional therapy, and local ablation (4-6). While surgical resection is considered the most common and effective method, there are also many difficulties in surgery, including the precise positioning of tumor tissue in the preoperative detection process and the precise resection of tumor mass in the surgical process.

In recent years, fluorescent contrast agent dye-guided surgery has developed vigorously in various fields of surgery. Clinically, fluorescent contrast agents can be used to accurately display tumor contours, guide surgery in real time, locate lymph node metastases, find micrometastasis, and identify important anatomical structures intraoperatively to avoid possible postoperative problems $(7,8)$. A fluorescence imaging surgical navigation system can locate tumor tissue more quickly and accurately during operation, 
greatly improving the success rate of surgery and reducing many postoperative adverse reactions (9). Indocyanine green (ICG) is a small organic near-infrared fluorescent dye of the anthocyanin family and the only near-infrared fluorescent dye approved by the FDA for clinical surgical use. After intravenous injection, it quickly binds to plasma proteins and is confined to the vascular lumen where it is absorbed before being metabolized by the liver and excreted in bile. The plasma clearance rate of ICG is high and its adverse reaction rate is less than $0.1 \%$ (10). The maximum absorption wavelength of ICG is around $800 \mathrm{~nm}$, and when combined with plasma protein it emits fluorescence with a peak wavelength of about $830 \mathrm{~nm}$ under near-infrared light irradiation, which easily penetrates tissues and cells and has a high ratio to background signals (11-13). In normal tissues, the endothelial space of microvessels is dense and structurally complete, while in solid tumor tissues, blood vessels are abundant, the vascular wall space is wide, and structural integrity is poor, resulting in selective high permeability and retention of macromolecules and lipid particles. This phenomenon is known as the enhanced permeability and retention effect (EPR) (14). After ICG binds to plasma proteins, the complex accumulates and targets the tumor region because of EPR, and the fluorescent signals generated by the excitation of nearinfrared light source are received by the imaging system and converted into visual images $(15,16)$. ICG-mediated fluorescence imaging navigation systems have been widely used in the resection of liver pancreatic, and breast cancer, and other clinical tumors $(17,18)$.

At present, there are many studies using fluorescence imaging systems to detect tumors, but the detection limit of fluorescence imaging systems is not yet clear. Our study first examined the fluorescence image surgery navigation system minimum visual concentration of ICG in vitro and the minimum detection limit of tumor in vivo. By establishing a tumor-burdened model involving ICG injection to the tail vein of mice, the system was used to detect the diameter of mouse axillary tumors, and the results were compared with vernier calipers to assess their detection limit and error, providing an experimental basis for the further development of digital diagnosis and treatment equipment in clinical practice. We present the following article in accordance with the ARRIVE reporting checklist (available at https:// dx.doi.org/10.21037/atm-21-3050).

\section{Methods}

\section{Mice and cell line}

We purchased 18-22 g female Institute of Cancer Research (ICR) mice from the Zhejiang Academy of Medical Sciences and Shanghai Jiesjie Laboratory Animal Co., Ltd., and 18-22 g female BALB/c Nude mice from the Changzhou Cavens Laboratory Animal Co., Ltd. Mice were housed under controlled room temperature $\left(22 \pm 2{ }^{\circ} \mathrm{C}\right)$ and humidity $(55 \% \pm 5 \%)$ on a $12 \mathrm{~h}$ light-dark cycle with free access to water and food in an SPF environment. Experiments were performed under a project license (No.: 2021-06013) granted by the committee of the Laboratory Animal Welfare in Pharmaceutical Animal Laboratory Center at China Pharmaceutical University, in compliance with the Laboratory Animal Welfare in Pharmaceutical Animal Laboratory Center guidelines for the care and use of animals.

Mouse sarcoma 180 cell line (S180) was purchased from the Jiangsu Cancer Drug Research Institute, and a human colon cancer cell line (HT-29) was purchased from the Shanghai Institute of Cells.

\section{Measurement of the lowest visible concentration in vitro}

A $0.0031 \mathrm{~g}$ sample of ICG was weighed and dissolved in $10 \mathrm{~mL}$ water for injection containing $10 \%$ fetal bovine serum (FBS) to prepare a solution of $4 \times 10^{-4} \mathrm{~mol} / \mathrm{L}$. This was then diluted to $1 \times 10^{-12} \mathrm{M}, 2 \times 10^{-12} \mathrm{M}, 4 \times 10^{-12} \mathrm{M}, 1 \times 10^{-11} \mathrm{M}$, $2 \times 10^{-11} \mathrm{M}, 4 \times 10^{-11} \mathrm{M}, 1 \times 10^{-10} \mathrm{M}, 2 \times 10^{-10} \mathrm{M}, 4 \times 10^{-10} \mathrm{M}$, $1 \times 10^{-9} \mathrm{M}, 2 \times 10^{-9} \mathrm{M}, 4 \times 10^{-9} \mathrm{M}$, and $0 \mathrm{M}$ proportionately with the injection water containing $10 \%$ FBS. These were placed in a 96-well plate, with three wells per concentration, and the final volume of ICG in each well was $200 \mu \mathrm{L}$. The spectral peak values of each well of the fixed spectral probe and the handheld spectral probe were read respectively, and the fluorescence brightness on the display screen was observed.

\section{Establishment of S180 tumor-burdened model mice}

The 120 female ICR mice were randomly divided according to body weight into a high-dose ICG solution group $(\mathrm{H}$, $4 \mathrm{mg} / \mathrm{kg}, 0.4 \mathrm{mg} / \mathrm{mL}$ ), medium-dose ICG solution group (M, $2 \mathrm{mg} / \mathrm{kg}, 0.2 \mathrm{mg} / \mathrm{mL}$ ), and low-dose ICG solution group (L, 
$1 \mathrm{mg} / \mathrm{kg}, 0.1 \mathrm{mg} / \mathrm{mL}$ ), with 40 mice in each group. According to the method of transplantation tumor study, solid tumors were inoculated under aseptic operation. The milky-white thick ascites of S180 ascites tumor mice were extracted and diluted into 1:5 cell suspension with normal saline, and each mouse was subcutaneously inoculated with $0.2 \mathrm{~mL}$ in the forelimb armpit. Tumor bearing mice in each group were injected with high, medium, and low doses of ICG $(0.2 \mathrm{~mL} / 20 \mathrm{~g})$ through the caudal vein at $0,1,2,3$, and 4 days after inoculation, and detected at $24 \mathrm{~h}$ after injection (19).

\section{Establishment of HT-29 tumor-burdened model mice}

Using the same method as described above, 120 female $\mathrm{BALB} / \mathrm{c}$ nude mice were grouped. According to the transplanted tumor study method, solid tumors were inoculated under aseptic procedures. Human colorectal cancer HT-29 cell lines in logarithmic growth phase were taken and prepared into a $5 \times 10^{6} / \mathrm{mL}$ cell suspension under aseptic conditions and $0.1 \mathrm{~mL}$ cell suspension was inoculated into the right axilla subcutaneously of BALB/c nude mice (20). The method of experiment after inoculation followed that described above.

\section{Tumor diameter test}

Within 24 hours of ICG injection, the mice were anesthetized by intraperitoneal injection of $2 \%$ chloral hydrate, and fixed. The skin at the inoculation site in the axilla was then cut to expose the solid tumor. The length and width of the solid tumor detected under the fluorescence imaging navigation system, were measured and recorded with a vernier caliper. Three mice in each group were randomly stripped of tumors and stained with hematoxylin-eosin (HE) (21).

\section{Statistical analysis}

ImageJ and SPSS 22.0 were used for data analysis and processing and all values were expressed as mean \pm SD.

\section{Results}

\section{Minimum visible concentration of ICG in vitro}

The concentrations of ICG respectively were $1 \times 10^{-12} \mathrm{M}$ (A1-A3), $2 \times 10^{-12} \mathrm{M}$ (A4-A6), $4 \times 10^{-12} \mathrm{M}$ (A7-A9), $1 \times 10^{-11} \mathrm{M}$ (B1-B3), $2 \times 10^{-11} \mathrm{M}$ (B4-B6), $4 \times 10^{-11} \mathrm{M}$ (B7-B9), $1 \times 10^{-10} \mathrm{M}$
(C1-C3), $2 \times 10^{-10} \mathrm{M}$ (C4-C6), $4 \times 10^{-10} \mathrm{M}$ (C7-C9), $1 \times 10^{-9} \mathrm{M}$ (D1-D3), $2 \times 10^{-9} \mathrm{M}$ (D4-D6), $4 \times 10^{-9} \mathrm{M}$ (D7-D9), and $0 \mathrm{M}$ (E1-E3). The green fluorescence of ICG detected by the fixed spectral probe at this concentration is shown in Figure 1, and the handheld spectral probe at this concentration is shown in Figure 2. As can be seen, the minimum visible concentration of ICG by the fixed spectral probe of the fluorescence imaging navigation system can reach $2 \times 10^{-11} \mathrm{M}$ (B4-B6), and by the handheld spectral probe can reach $1 \times 10^{-12} \mathrm{M}(\mathrm{A} 1-\mathrm{A} 3)$.

\section{Minimum tumor diameter}

HE staining results shows fibrous connective tissue within the article, funicular and small nests of epithelioid cells, and bright or eosinophilic cytoplasm, and the cell nuclei have obvious atypia, obvious nucleoli, nuclear fission, and apoptosis necrosis (Figures 3,4). The histological appearance of the tumor is consistent.

As shown in Tables 1,2 and Figures 5,6, the mean values of measured tumor length and width increased with the increase of inoculation days, and green fluorescence is seen in the axillary inoculation site. The accuracy of the fluorescence imaging navigation system is high, with the minimum detection limit up to $0.2 \mathrm{~mm}$, and the measured error between the system and vernier calipers is within $0.2 \mathrm{~mm}$.

\section{Discussion}

Cancer is the leading cause of death in developed countries and the second in developing countries $(22,23)$. As surgery is the main treatment for many malignant tumors, precise localization of the tumor resection area plays a crucial role in the prognosis of patients (9). Currently, the main clinical imaging examinations for localization include computed tomography (CT), magnetic resonance imaging (MRI), positron emission tomography/CT (PET/CT), and single photon emission computed tomography (SPECT/CT) (24). However, the above detection methods are often used for preoperative diagnosis and postoperative evaluation, which have little significance for the accurate location of the intraoperative tumor margin. As it is noninvasive, realtime, sensitive and inexpensive, and can be used to achieve tissue, cellular and subcellular imaging of living animals by bioluminescence or fluorescence (25), the development of optical molecular image navigation technology has provided a new option for intraoperative tumor visualization. Fluorescent surgical navigation technology 

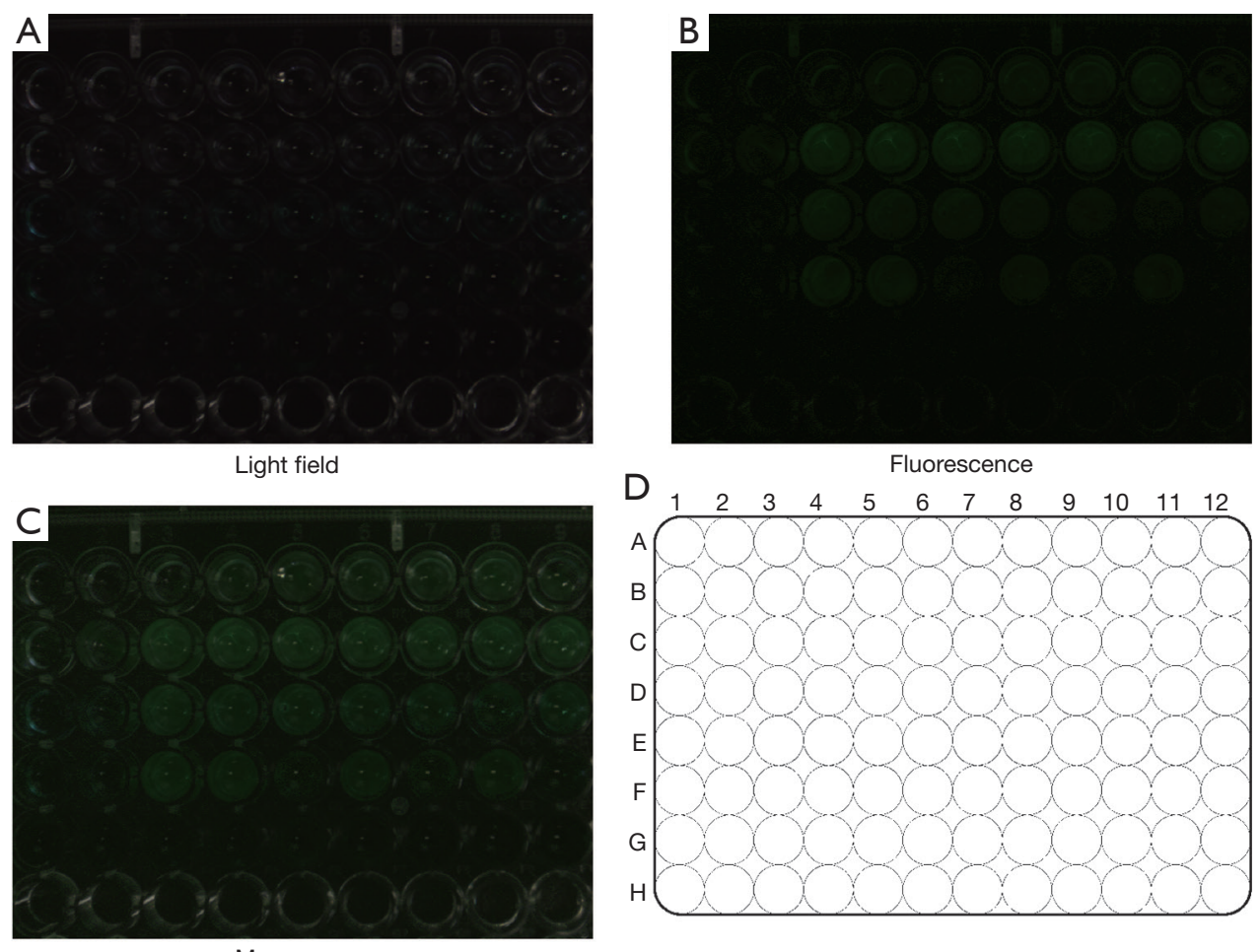

Merge

Figure 1 The fluorescence intensity of ICG solutions with different concentrations under the fixed spectral probe of the fluorescent imaging surgical navigation system. (A) Bright field pictures of ICG at different concentrations; (B) fluorescence images of ICG at different concentration; (C) merge of bright field and fluorescence composite images; (D) 96-well plate distribution diagram. ICG, indocyanine green.

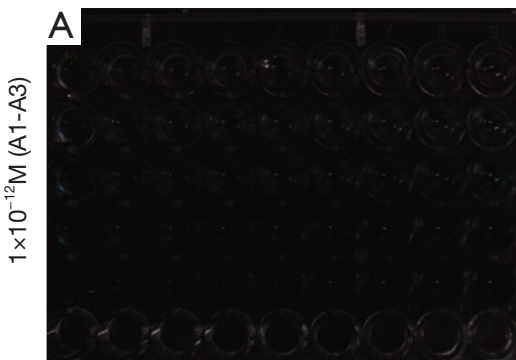

Light field

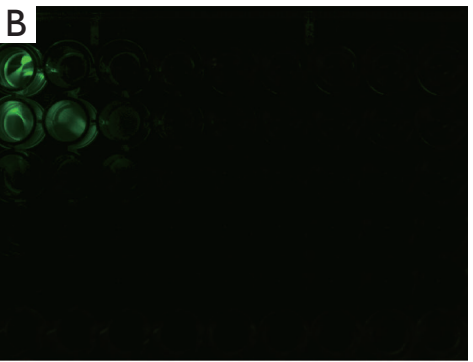

Fluorescence

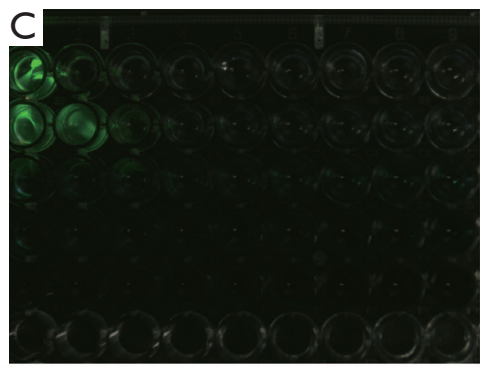

Merge

Figure 2 The fluorescence intensity of ICG solutions with different concentrations under the handheld spectral probe of the fluorescent imaging surgical navigation system. (A) Bright field pictures of ICG at different concentrations; (B) fluorescence images of ICG at different concentration; (C) merge of bright field and fluorescence composite images. ICG, indocyanine green.

has been widely used in sentinel lymph node mapping, solid tumor identification, lymphography, angiography, and intraoperative anatomical imaging. At present, most intraoperative fluorescence navigation systems are equipped with near-infrared sensing detectors and charge coupled devices cameras. Compared with visible light, near-infrared light is a common light source for optical molecular imaging because of its strong penetrating ability, less autofluorescence, and higher signal-to-noise ratio (26). ICG is the only nearinfrared fluorescent dye currently approved by the FDA for 


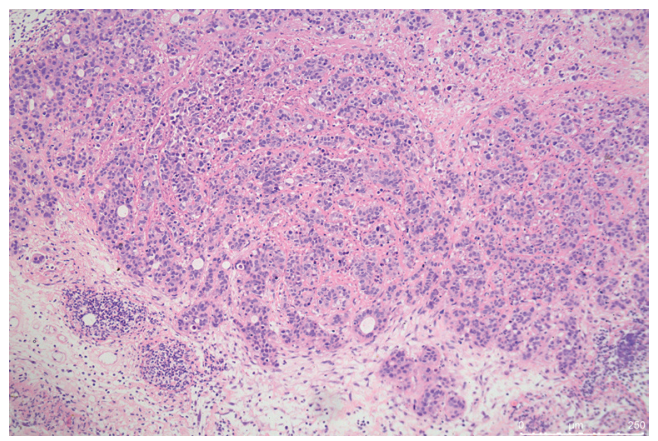

Figure 3 The HE staining diagram of HT-29 solid tumor (100x) showed that the tumor cells were densely arranged in bands and clusters, with disordered arrangement. The phenomenon of nuclear mitosis was common, and locally the cells were arranged in a ring to form a glandular cavitary structure. HE, hematoxylin-eosin.

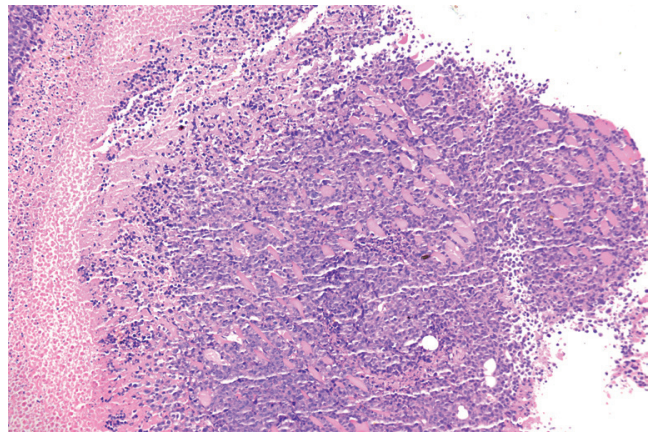

Figure 4 Representation of HE staining for solid tumor of S180 $(100 \times)$. The tumor cells were densely cabled and clumped, with different cell sizes and shapes, obvious atypia, and frequent nuclear mitosis. HE, hematoxylin-eosin.

Table 1 Size of S180 solid tumor (mm, mean \pm SD, $n=8)$

\begin{tabular}{|c|c|c|c|c|c|c|}
\hline Group & Value (mm) & \multicolumn{5}{|c|}{ Time of injection after inoculation } \\
\hline \multirow[t]{3}{*}{ High dose (4 mg/kg) } & Fluorescence length & $0.51 \pm 0.17$ & $1.65 \pm 0.18$ & $1.91 \pm 0.17$ & $4.50 \pm 0.76$ & $5.23 \pm 1.05$ \\
\hline & Fluorescence wide & $0.28 \pm 0.11$ & $1.32 \pm 0.25$ & $1.36 \pm 0.25$ & $2.80 \pm 1.10$ & $3.19 \pm 0.36$ \\
\hline & Virtual length & $0.40 \pm 0.23$ & $1.76 \pm 0.12$ & $1.68 \pm 0.13$ & $4.52 \pm 0.93$ & $5.23 \pm 1.18$ \\
\hline \multirow[t]{4}{*}{ Middle dose $(2 \mathrm{mg} / \mathrm{kg})$} & Fluorescence length & $0.3 \pm 0.24$ & $1.73 \pm 0.29$ & $2.09 \pm 0.36$ & $4.23 \pm 0.45$ & $5.80 \pm 0.97$ \\
\hline & Fluorescence wide & $0.29 \pm 0.25$ & $1.55 \pm 0.25$ & $1.36 \pm 0.23$ & $2.90 \pm 0.67$ & $3.85 \pm 0.75$ \\
\hline & Virtual length & $0.45 \pm 0.38$ & $1.72 \pm 0.25$ & $1.73 \pm 0.27$ & $4.15 \pm 0.50$ & $5.68 \pm 1.07$ \\
\hline & Virtual wide & $0.25 \pm 0.25$ & $1.52 \pm 0.25$ & $1.32 \pm 0.21$ & $2.95 \pm 0.64$ & $3.82 \pm 0.83$ \\
\hline \multirow{2}{*}{ Low dose (1 mg/kg) } & Virtual length & $0.56 \pm 0.35$ & $1.49 \pm 0.22$ & $1.56 \pm 0.24$ & $4.24 \pm 0.71$ & $6.27 \pm 1.24$ \\
\hline & Virtual wide & $0.37 \pm 0.26$ & $1.22 \pm 0.17$ & $1.41 \pm 0.13$ & $3.15 \pm 0.79$ & $4.27 \pm 0.92$ \\
\hline
\end{tabular}

clinical surgical use. Some studies pointed out that ICGguided fluorescence intraoperative navigation systems can detect small tumor lesions of $2-5 \mathrm{~mm}$, but clinical tumor resection has higher requirements for precise identification and accuracy of tumor edge recognition ICG (27).

This study first ascertained the ICG minimum concentration of the fluorescence image surgery navigation system in vitro. Through the establishment of a tumorburdened mice model, we explored the minimum detection limit of the in vivo fluorescence intraoperative navigation system, which provided an experimental basis for the further development of optical molecular imaging technology in the field of clinical tumor resection. In this experiment, the inoculation site of tumor-bearing mice was exposed and placed under the fixed probe about $24 \mathrm{~h}$ after ICG injection, at which point green fluorescence from the tumor site could be seen, and the fluorescence range increased with the growth of the tumor. The minimum detection limit was up to $0.2 \mathrm{~mm}$. The minimum detection concentration of ICG in vitro with the fixed spectral probe of the fluorescent 
Table 2 Size of HT-29 solid tumor (mm, mean \pm SD, $\mathrm{n}=8$ )

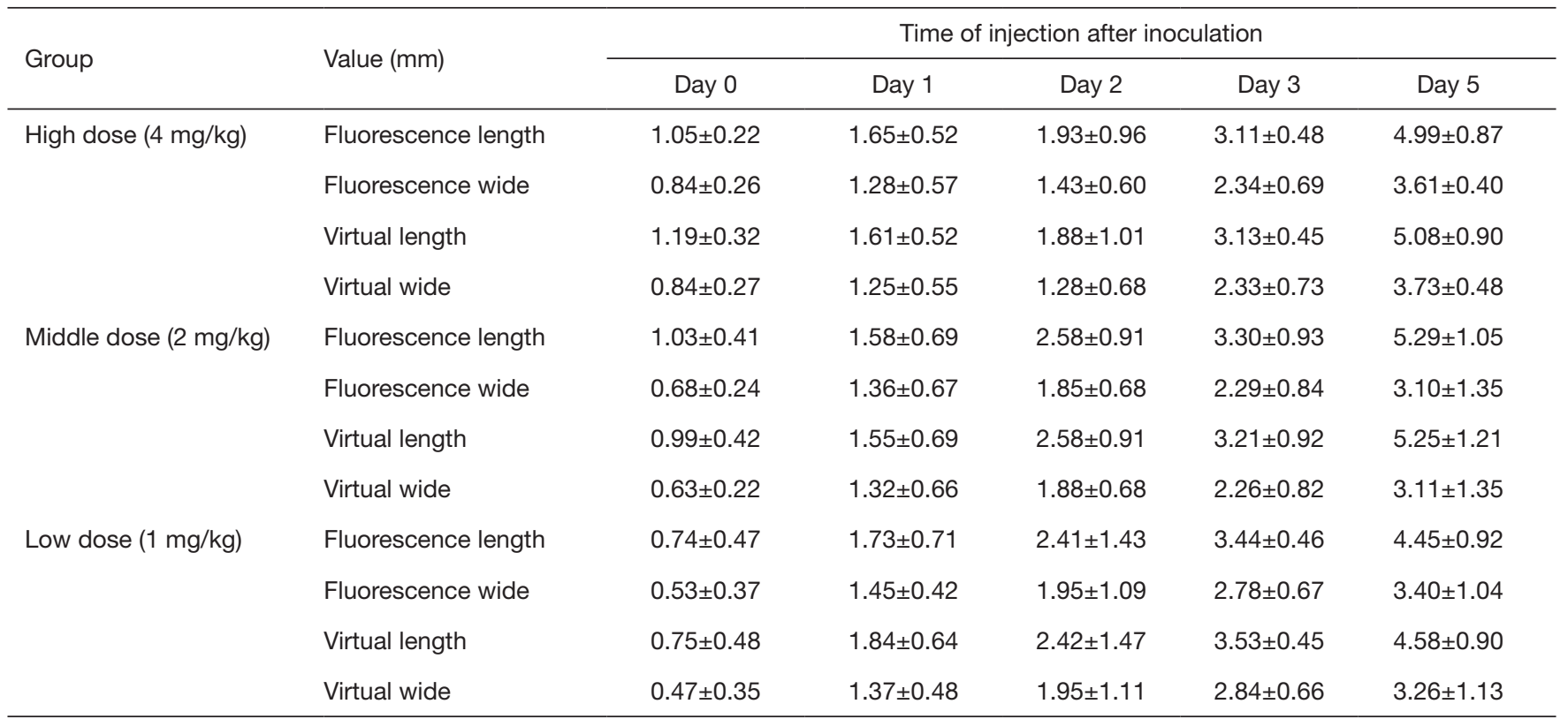

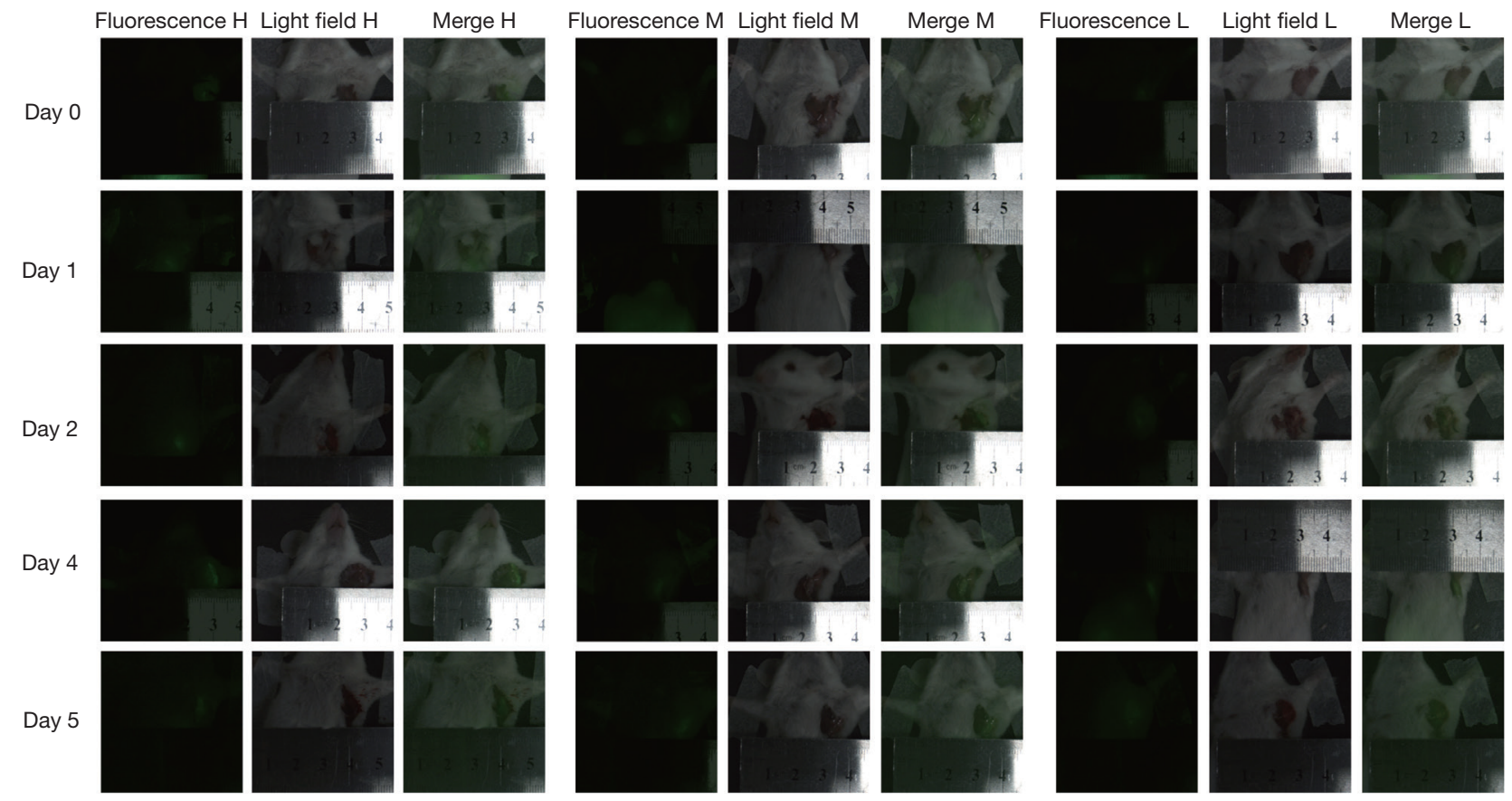

Figure 5 Representative images of each group of S180 solid tumor detected by surface light source using fluorescent imaging surgical navigation system. Representative images of fluorescence images, bright field images, and merge images of each group of animals under fixed spectral probe $0-5$ days after inoculation of S180 solid tumor. H: high-dose (4 mg/ $\mathrm{kg})$ group of ICG solution; M: middle-dose (2 mg/kg) group of ICG solution; L: low-dose $(1 \mathrm{mg} / \mathrm{kg})$ group of ICG solution. ICG, indocyanine green. 


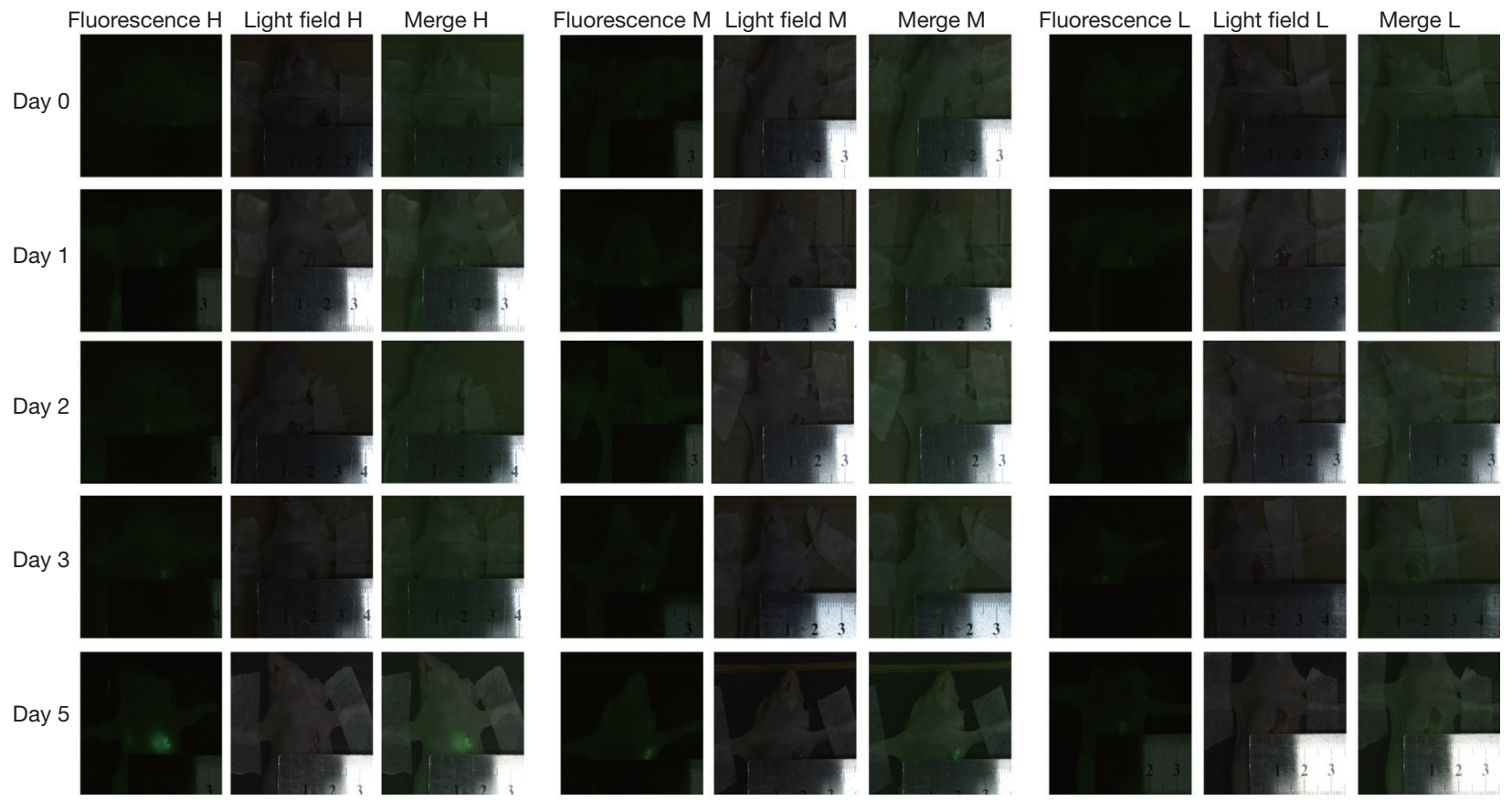

Figure 6 Representative images of each group of HT-29 solid tumor detected by surface light source using fluorescent imaging surgical navigation system. Representative images of fluorescence images, bright field images and merge images of each group of animals under fixed spectral probe $0-5$ days after inoculation of HT-29 solid tumor. H: high-dose ( $4 \mathrm{mg} / \mathrm{kg}$ ) group of ICG solution; M: middle-dose ( $2 \mathrm{mg} / \mathrm{kg}$ ) group of ICG solution; L: low-dose $(1 \mathrm{mg} / \mathrm{kg})$ group of ICG solution. ICG, indocyanine green.

imaging surgical navigation system was up to $2 \times 10^{-11} \mathrm{M}$, and that with the handheld spectral probe was up to $1 \times 10^{-12} \mathrm{M}$. The above results indicate that, compared with the traditional visual identification of the tumor margin, the fluorescent imaging surgical navigation system has higher sensitivity and accuracy, which is of great significance for the accurate identification and resection of the tumor margin and the improvement of prognosis.

There are also some limitations in the application of fluorescence imaging navigation systems. Currently, the main challenge is the development of tumor specific targeting fluorescence contrast agents with high fluorescence efficiency (28). New fluorescent probes, such as monoclonal antibody coupled fluorescent probes, cell surface enzyme activated fluorescent probes, and lysosomal activated fluorescent probes, are better than traditional fluorescent probes such as ICG, methylene blue, and sodium fluorescein in tumor targeting and background signal ratio. However, their safety remains to be established and their clinical transformation faces certain difficulties $(29,30)$. With the continuous improvement and development of technology, more targeted fluorescent imaging probes have been developed, providing great potential for the application of fluorescent imaging surgical navigation technology to reduce postoperative recurrence rate and improve postoperative survival in the future.

\section{Acknowledgments}

Funding: This work was supported by the National Key Research and Development Program of China (2016YFC0104100) and Key Promotion Project of Core Technology of Jiangsu Province (2018).

\section{Footnote}

Reporting Checklist: The authors have completed the ARRIVE reporting checklist. Available at https://dx.doi. org/10.21037/atm-21-3050

Data Sharing Statement: Available at https://dx.doi. org/10.21037/atm-21-3050

Conflicts of Interest: All authors have completed the ICMJE 
uniform disclosure form (available at https://dx.doi. org/10.21037/atm-21-3050). HC and YW are employees of Nanjing Nuoyuan Medical Devices Co., Ltd., Nanjing, China. The other authors have no conflicts of interest to declare.

Ethical Statement: The authors are accountable for all aspects of the work in ensuring that questions related to the accuracy or integrity of any part of the work are appropriately investigated and resolved. Experiments were performed under a project license (No.: 2021-06013) granted by the committee of the Laboratory Animal Welfare in Pharmaceutical Animal Laboratory Center at China Pharmaceutical University, in compliance with the Laboratory Animal Welfare in Pharmaceutical Animal Laboratory Center guidelines for the care and use of animals.

Open Access Statement: This is an Open Access article distributed in accordance with the Creative Commons Attribution-NonCommercial-NoDerivs 4.0 International License (CC BY-NC-ND 4.0), which permits the noncommercial replication and distribution of the article with the strict proviso that no changes or edits are made and the original work is properly cited (including links to both the formal publication through the relevant DOI and the license). See: https://creativecommons.org/licenses/by-nc-nd/4.0/.

\section{References}

1. Bray F, Ferlay J, Soerjomataram I, et al. Global cancer statistics 2018: GLOBOCAN estimates of incidence and mortality worldwide for 36 cancers in 185 countries. CA Cancer J Clin 2018;68:394-424. Erratum in: CA Cancer J Clin 2020;70:313.

2. GBD 2017 Disease and Injury Incidence and Prevalence Collaborators. Global, regional, and national incidence, prevalence, and years lived with disability for 354 diseases and injuries for 195 countries and territories, 1990-2017: a systematic analysis for the Global Burden of Disease Study 2017. Lancet 2018;392:1789-858. Erratum in: Lancet 2019;393:e44.

3. Carrera PM, Kantarjian HM, Blinder VS. The financial burden and distress of patients with cancer: understanding and stepping-up action on the financial toxicity of cancer treatment. CA Cancer J Clin 2018;68:153-65.

4. Mahara G, Chen G, Ge Q, et al. A comprehensive intervention on feasibility, efficacy, and safety between
TAE combined with multi-applicator ablation therapy and TACE in the treatment of large hepatocellular carcinoma. Transl Cancer Res 2020;9:6303-12.

5. Gotwals P, Cameron S, Cipolletta D, et al. Prospects for combining targeted and conventional cancer therapy with immunotherapy. Nat Rev Cancer 2017;17:286-301.

6. Le Fevre C, Waissi W, Chambrelant I, et al. A critical narrative review of radiotherapy for retroperitoneal soft tissue sarcoma. Chin Clin Oncol 2020;9:79.

7. Porcu EP, Salis A, Gavini E, et al. Indocyanine green delivery systems for tumour detection and treatments. Biotechnol Adv 2016;34:768-89.

8. Huang $\mathrm{X}, \mathrm{Wu} \mathrm{J}, \mathrm{He} \mathrm{M}$, et al. Combined cancer chemophotodynamic and photothermal therapy based on ICG/PDA/TPZ-loaded nanoparticles. Mol Pharm 2019;16:2172-83.

9. Ishizawa T, Fukushima N, Shibahara J, et al. Real-time identification of liver cancers by using indocyanine green fluorescent imaging. Cancer 2009;115:2491-504.

10. Alander JT, Kaartinen I, Laakso A, et al. A review of indocyanine green fluorescent imaging in surgery. Int $\mathrm{J}$ Biomed Imaging 2012;2012:940585.

11. Landsman ML, Kwant G, Mook GA, et al. Lightabsorbing properties, stability, and spectral stabilization of indocyanine green. J Appl Physiol 1976;40:575-83.

12. Hernot $S$, van Manen L, Debie P, et al. Latest developments in molecular tracers for fluorescence imageguided cancer surgery. Lancet Oncol 2019;20:e354-67.

13. Jiang JX, Keating JJ, Jesus EM, et al. Optimization of the enhanced permeability and retention effect for nearinfrared imaging of solid tumors with indocyanine green. Am J Nucl Med Mol Imaging 2015;5:390-400.

14. Maeda H, Nakamura H, Fang J. The EPR effect for macromolecular drug delivery to solid tumors: improvement of tumor uptake, lowering of systemic toxicity, and distinct tumor imaging in vivo. Adv Drug Deliv Rev 2013;65:71-9.

15. Alacam B, Yazici B, Intes X, et al. Pharmacokinetic-rate images of indocyanine green for breast tumors using nearinfrared optical methods. Phys Med Biol 2008;53:837-59.

16. Wang C, Wang Z, Zhao T, et al. Optical molecular imaging for tumor detection and image-guided surgery. Biomaterials 2018;157:62-75.

17. Namikawa T, Sato T, Hanazaki K. Recent advances in near-infrared fluorescence-guided imaging surgery using indocyanine green. Surg Today 2015;45:1467-74.

18. Lau CT, Au DM, Wong KKY. Application of indocyanine green in pediatric surgery. Pediatr Surg Int 
2019;35:1035-41.

19. Zou S, Duan B, Xu X. Inhibition of tumor growth by $\beta$-glucans through promoting CD4+ T cell immunomodulation and neutrophil-killing in mice. Carbohydr Polym 2019;213:370-81.

20. Chitchumroonchokchai C, Thomas-Ahner JM, Li J, et al. Anti-tumorigenicity of dietary $\alpha$-mangostin in an HT-29 colon cell xenograft model and the tissue distribution of xanthones and their phase II metabolites. Mol Nutr Food Res 2013;57:203-11.

21. Kather JN, Krisam J, Charoentong P, et al. Predicting survival from colorectal cancer histology slides using deep learning: a retrospective multicenter study. PLoS Med 2019; 16:e1002730.

22. Jemal A, Bray F, Center MM, et al. Global cancer statistics. CA Cancer J Clin 2011;61:69-90. Erratum in: CA Cancer J Clin 2011;61:134.

23. GBD 2019 Risk Factors Collaborators. Global burden of 87 risk factors in 204 countries and territories, 1990-2019: a systematic analysis for the Global Burden of Disease Study 2019. Lancet 2020;396:1223-49.

24. Sun SJ, Feng YY, Zhang Y, et al. Antitumor and

Cite this article as: Zhao $\mathrm{X}$, Wang $\mathrm{Z}, \mathrm{Wu} \mathrm{Y}$, Cai $\mathrm{H}$. Application of an indocyanine green-mediated fluorescence imaging navigation system in detecting mice tumors. Ann Transl Med 2021;9(15):1238. doi: 10.21037/atm-21-3050 immunoregulatory activities of seleno- $\beta$-lactoglobulin on S180 tumor-bearing mice. Molecules 2017;23:46.

25. Luo S, Zhang E, Su Y, et al. A review of NIR dyes in cancer targeting and imaging. Biomaterials 2011;32:7127-38.

26. Gioux S, Choi HS, Frangioni JV. Image-guided surgery using invisible near-infrared light: fundamentals of clinical translation. Mol Imaging 2010;9:237-55.

27. Satou S, Ishizawa T, Masuda K, et al. Indocyanine green fluorescent imaging for detecting extrahepatic metastasis of hepatocellular carcinoma. J Gastroenterol 2013;48:1136-43.

28. Sheng Z, Hu D, Xue M, et al. Indocyanine green nanoparticles for theranostic applications. Nano-Micro Letters 2013;5:145-50.

29. Rosenthal EL, Warram JM, Bland KI, et al. The status of contemporary image-guided modalities in oncologic surgery. Ann Surg 2015;261:46-55.

30. Nagaya T, Nakamura YA, Choyke PL, et al. Fluorescenceguided surgery. Front Oncol 2017;7:314.

(English Language Editor: B. Draper) 